tain several fragments, all of which apparently were able to reassociate with the protein. The ability of so small a protein molecule to protect so vast a length of RNA is extraordinary and unexplained.

Now Zimmerman et al. (Proc. US Nat. Acad. Sci., 69, 1282; 1972) have varied this approach and extended it to the other primary binding proteins, and have gone some way towards localizing the six binding sites on the 16S RNA. They have prepared nuclease fragments of the RNA and tested them for ability to bind the proteins. $\mathrm{T}_{1}$ RNA generates initially two large fragments of $12 \mathrm{~S}$ and $8 \mathrm{~S}$, which comprise the greater part of the whole chain. With further digestion the $12 \mathrm{~S}$ piece gives place to $10 \mathrm{~S}$ and $4 \mathrm{~S}$ fragments. Pancreatic ribonuclease, on the other hand, produces an $11 \mathrm{~S}$ fragment, which in turn generates two smaller segments of $9 S$ and $4 S$. Five of the six proteins bind to the $12 \mathrm{~S}$ fragment, whereas one (S13) binds to the 8S. Nucleotide mapping shows that the $12 S$ piece is essentially the $5^{\prime}$ half of the 16S RNA, and the 8S adjoins it on the $3^{\prime}$ side. The $4 \mathrm{~S}$ fragment, which has 135 nucleotides, contains a site for only one of the proteins (S15). The S4 protein of Schaup et al. is the only one to bind to the pancreatic 9S segment, which comes from the $5^{\prime}$ end of the $12 \mathrm{~S}$ and therefore of the original 16S RNA.

The precise location of the fragments within the $16 \mathrm{~S}$ chain is limited by the incompleteness of the sequence data so far available. The conclusion is, however, that five of the six primary binding sites are clustered rather closely together in one half of the chain. Moreover, a large part of the 4S RNA, which binds the protein $\$ 15$, has been sequenced, and also partial sequence information for the $9 S$ fragment, that binds S4, is presented. As Schaup et al. found, this fragment consists of several smaller pieces, which separate under denaturing conditions. In both cases there seems to be considerable scope for the formation of hairpin loops, with considerable base-pairing, but the precise relation of this to the RNA-protein interaction must await the isolation of smaller binding fragments-a project which Zimmerman et al. assure readers is in progress.

At the next, higher, level of ribosome assembly, protein-protein interactions come into play, and a good deal of effort is now being deployed on attempts to establish which proteins interact with which. A number of reports have appeared of the isolation of large fragments of the ribosome that contain some proteins and not others, and so allow the possible range of protein-protein contacts to be narrowed down. Most encouragingly perhaps, Schendel et al. (ibid., 544) were able, by hewing the $30 \mathrm{~S}$ subunit into three pieces with nuclease, and identifying the proteins distributed between them, to relate the interactions which are compatible with this distribution to those dictated by Nomura's assembly maps. That is to say, those proteins, the incorporation of which into the $30 \mathrm{~S}$ ribosome is contingent on the prior presence of the primary binding proteins on the RNA, are also present in the same nucleolytic subfragment as the latter.

This suggests that the assembly maps reflect direct physical interaction of the proteins which they link, rather than a merely conformation-directing role for the primary binding proteins. Bickle et al. (ibid., 1327) have found that the action of a bifunctional reagent on $30 \mathrm{~S}$ subunits leads apparently to the pairwise association of some of the proteins, but the information that emerges is insufficient to allow comparisons with the assembly maps. The interaction of the more readily dissociable proteins has also been studied, and Randall-Hazelbauer and Kurland (Mol. Gen. Genet., 115, 234; 1972) have found that the binding of tRNA to ribosomes is enhanced by augmentation of three proteins, S2, S3 and S14 (S1 being already known to promote messenger binding). These components may therefore well constitute the A-site of the $30 \mathrm{~S}$ ribosome.

The first step in identifying the interaction region on the 23S RNA for the 5S minor RNA component has been taken by Gray and Monier (Biochimie, 54, 41 ; 1972). Earlier work from the same laboratory showed that a small group of large subunit proteins was required for the formation of a complex containing $5 S$ and 23S RNA. Four proteins have in fact been identified as necessary and sufficient. Gray and Monier now find that the same trick is possible with a fragment of the 23S RNA, comprising about 60 per cent of the whole, and lying at the $3^{\prime}$ end. Attempts will no doubt be made to repeat the experiment with smaller pieces.

\section{IMMUNE RESPONSE \\ Genetic Control}

from a Correspondent

A CONFERENCE on the genetic control of immune responsiveness: its relationship to disease susceptibility at Brook Lodge, Michigan, on May 8-10 showed how rapid developments in this field have greatly clarified certain issues but have left others obscure.

Studies with recombinant mice during the past two years carried out by $\mathrm{Dr}$ D. C. Schressler (Ann Arbor) have confirmed that there are two

\title{
SV40 Induces Nuclear Acidic Protein Synthesis
}

THE small DNA tumour virusespolyoma virus and simian virus 40 . share the property of inducing nondividing cells which they infect to initiate a round of DNA synthesis; if the cells are non-permissive and survive, they subsequently mitose.

This triggering of DNA replication by polyoma virus and SV40 some 12 hours after infection involves all the ccllular DNA, mitochondrial as well as nuclear, and in the nucleus it is accompanied by the induction of the synthesis of histone proteins required for the daughter chromosomes. But the chromosomes of mammalian cells contain considerable proportions of acidic proteins in addition to the basic histones. Is the synthesis of this class of nuclear proteins also induced after infection by polyoma virus or SV40?

The results of experiments which Rovera, Baserga and Defendi report in next Wednesday's Nature New Biology (June 21) indicate that in both permissive monkey CV1 cells and non-permissive secondary Chinese hamster fibroblasts synthesis of nuclear acidic proteins is indeed induced by SV40 infection. That may be entirely as expected, but what is interesting is that the induction occurs within 3 hours of infection, well before the synthesis of DNA and histones is induced.

Using incorporation of ${ }^{3} \mathrm{H}$-leucine into acid insoluble material as an index of protein synthesis, Rovera et al. measured the synthesis of total cytoplasmic protein and of acidic nuclear proteins in CV1 cells and Chinese hamster cells 3 hours after infection or mock infection. At the multiplicities of infection they used, essentially all the cells were infected In both infected permissive and non-permissive cells, while the rate of incorporation of ${ }^{3} \mathrm{H}$ leucine into cytoplasmic proteins decreases, the rate of synthesis of acidic nuclear proteins increases.

Precisely how, within 3 hours of infection, SV40 achieves this differential stimulation of the synthesis of host cell proteins is puzzling, but as Rovera $e t$ al. point out, by 3 hours after infection at least some SV40 particles have been uncoated and so the synthesis of viral proteins, including the trigger molecule, may have occurred. They also comment that the similarities between the pattern of synthesis of acidic nuclear proteins in cells stimulated to divide by SV40 or by serum are sufficient reasonably to suggest that both stimuli act through a common pathway. 